\title{
Excess mortality, causes of death and prognostic factors in anorexia nervosa
}

Fotios C. Papadopoulos, Anders Ekbom, Lena Brandt and Lisa Ekselius

\section{Background}

Anorexia nervosa is a mental disorder with high mortality.

\section{Aims}

To estimate standardised mortality ratios (SMRS) and to investigate potential prognostic factors.

\section{Method}

Six thousand and nine women who had in-patient treatment for anorexia nervosa were followed-up retrospectively using Swedish registers

\section{Results}

The overall SMR for anorexia nervosa was 6.2 (95\% Cl 5.57.0). Anorexia nervosa, psychoactive substance use and suicide had the highest SMR. The SMR was significantly increased for almost all natural and unnatural causes of death. The SMR 20 years or more after the first hospitalisation remained significantly high. Lower mortality was found during the last two decades. Younger age and longer hospital stay at first hospitalisation was associated with better outcome, and psychiatric and somatic comorbidity worsened the outcome.

\section{Conclusions}

Anorexia nervosa is characterised by high lifetime mortality from both natural and unnatural causes. Assessment and treatment of psychiatric comorbidity, especially alcohol misuse, may be a pathway to better long-term outcome.

\section{Declaration of interest}

None. Funding detailed in Acknowledgements.
Anorexia nervosa is a serious mental disorder seen mostly in teenaged girls and young women. Mortality in anorexia nervosa has been investigated extensively. However, reported standardised mortality ratios (SMRs) vary substantially from 0 to 17.8 (Table 1 and online Table DS1). ${ }^{1-24}$ Standardised mortality ratio estimates for specific causes of death were only reported in one populationbased study, with inconclusive results. ${ }^{21}$ Other studies reported an increased SMR for suicide 3,25 and mainly non-significant, decreased SMR for cancer. ${ }^{21,26,27}$ Although psychiatric comorbidity in anorexia has been investigated in several studies, the extent to which it can affect mortality is unclear. The primary aim of this study was to assess mortality, both from natural and unnatural causes, in a nationwide cohort of people with anorexia nervosa and to compare findings with the general population. A secondary aim was to investigate potential prognostic factors for the fatal outcome of anorexia.

\section{Method}

\section{Swedish registers}

The Swedish Cause-of-Death Register ${ }^{28}$ includes all individuals who died either in Sweden or abroad since 1952 and who were registered in Sweden at the time of death. The statistics do not include stillborns, people who died on a temporary visit to Sweden or asylum seekers who had not yet obtained residence permits. Those who have emigrated and are no longer registered in Sweden are also not included. The data are based on death certificates that provide information on date as well as underlying (or main) and secondary causes of death using the ICD. All external causes of death (homicides, suicides, accidents), were considered as unnatural causes of death, whereas every other cause of death was considered as a natural cause. Anorexia nervosa in this context was considered as a natural cause of death.

The Swedish Hospital Discharge Register ${ }^{29}$ covers all in-patient hospitalisations in both somatic and psychiatric settings in Sweden. It was founded in 1965 and has included the entire Swedish population since 1987. Each record includes the patient's
National Registration Number (NRN) - a unique personal identifier assigned to all Swedish residents - admission and discharge dates, the main discharge diagnosis and up to seven secondary diagnoses. There are almost no private in-patient facilities in Sweden and the Hospital Discharge Register is therefore population-based.

The Swedish Cancer Register contains all diagnosed cancer cases among Swedish residents since 1 January 1958. The Cancer Register was used in order to find those individuals with a cancer diagnosis at the first anorexia nervosa admission.

\section{Definitions and study population}

Patients discharged from hospital during the period from 1 January 1973 to 31 December 2003, with a main or secondary diagnosis of anorexia nervosa were identified in the Swedish Hospital Discharge Register using the following ICD codes: 306.50 (ICD-8 from 1973 to 1986), ${ }^{30} 307 \mathrm{~B}$ (ICD-9 from 1987 to 1996$)^{31}$ and F50.0 or F50.1 (ICD-10 from 1997 to 2003). ${ }^{32}$ Only females aged 10-40 at the time of discharge were included. This age limit was utilised in order to eliminate diagnostic misclassification (e.g. feeding complications in children or older individuals). For the same reason, 55 women who already had a cancer diagnosis before the first hospitalisation for anorexia were excluded. The follow-up time for those individuals who died from natural causes started 1 year after the first hospitalisation, and 14 women who died from natural causes during the first year after the first discharge with an anorexia nervosa diagnosis were excluded. Thus, there is a reduced risk of a diagnostic misclassification of individuals with cancer or with other severe somatic diseases as having anorexia.

History and comorbidity of somatic and psychiatric disorders are based upon data on in-patient care for either somatic or psychiatric diseases other than anorexia nervosa. An individual was considered to have a comorbid psychiatric disorder if she also received separate in-patient treatment for that disorder. Similarly, for an individual to be considered to have a comorbid somatic 
Table 1 Studies that report standardised mortality ratios (SMRS) in eating disorders by descending SMR. ${ }^{a}$ (For a more detailed version, see online Table DS1)

\begin{tabular}{|c|c|c|c|c|c|}
\hline Study & $n$ & $\begin{array}{l}\text { Follow-up } \\
\text { Years, mean }\end{array}$ & Diagnostic system & Crude MR, \% & SMR (95\% Cl) \\
\hline Norring \& Sohlberg, $1993^{1}$ & 48 & 6.0 & DSM-III-R & 6.3 & $17.8(3.7-51.9)^{\mathrm{d}}$ \\
\hline Eckert et al, $1995^{2}$ & 76 & 9.6 & DSM-III-R & 6.6 & $12.8(4.2-29.9)^{\mathrm{d}}$ \\
\hline Keel et al, $2003^{3}$ & 136 & 8.6 & DSM-IV & 7.4 & $11.6(5.5-21.3)$ \\
\hline Lee et al, $2003^{4}$ & 88 & 9.0 & DSM-III-R & 3.4 & $10.5(2.2-30.8)^{\mathrm{d}}$ \\
\hline Birmingham et al, $2005^{5}$ & 326 & 7.3 & DSM-III/ICD-10 & 5.2 & $10.5(5.5-15.5)$ \\
\hline Lowe et al, $2001^{6}$ & 84 & 21.3 & DSM-IV & 16.7 & 9.8 \\
\hline Signorini et al, $2007^{7}$ & 147 & $8^{c}$ & DSM-IV & 2.7 & 9.7 \\
\hline Moller-Madsen et al, $1996^{8}$ & 790 & 7.8 & ICD-8 & 5.7 & $9.2(6.7-12.3)$ \\
\hline Fichter et al, $2006^{9}$ & 103 & $12.0^{\mathrm{C}}$ & DSM-IV & 6.8 & $8.9(2.3-15.4)$ \\
\hline Crow et al, $1999^{10}$ & 54 & 7.5 & Undefined & 7.4 & 8.4 \\
\hline Lindblad et al, $2006^{11}$ & 564 & $12-16^{\mathrm{C}}$ & ICD-8 & 4.4 & 7.7 \\
\hline Joergensen, $1992^{12}$ & $132^{\mathrm{b}}$ & 11.7 & DSM-III-R & 16.7 & $7.2(4.5-10.8)$ \\
\hline Pagsberg \& Wang, $1994^{13}$ & $50^{b}$ & 6.2 & ICD-10 & 6 & $6.9(1.4-20.2)$ \\
\hline Emborg, $1999^{14}$ & 2763 & 10.3 & ICD-8 & 8.4 & $6.7(5.7-7.8)$ \\
\hline Patton, $1988^{15}$ & 332 & 7.6 & Russel (1970) & 3.3 & $6.0(3.0-10.8)^{d}$ \\
\hline Tolstrup et al, $1985^{16}$ & 151 & 22.9 & Tolstrup (1985) & 11.9 & $4.8(2.6-7.6)$ \\
\hline Crisp et al, $1992^{17}$ & $63^{b}$ & 22.1 & DSM-III-R & 13.0 & $4.7(2.0-9.3)^{d}$ \\
\hline Millar et al, $2005^{18}$ & $487^{b}$ & Up to $35^{c}$ & Aberdeen, ICD-9 & 4.1 & $3.2(2.1-5.0)$ \\
\hline Theander, $1970^{19}$ & 94 & 36.2 & Theander (1970) & 25.5 & $2.9(1.9-4.4)$ \\
\hline Lindblad et al, 2006 $6^{11}$ & 554 & $12-16^{\mathrm{C}}$ & ICD-9 & 1.3 & 2.9 \\
\hline Crisp, $2006^{20}$ & 850 & $0-30^{c}$ & DSM-IV & 5.5 & $1.0(0.8-1.3)$ \\
\hline Korndorfer et al, $2003^{21}$ & $208^{b}$ & 27.1 & DSM-III-R & 8.2 & $0.7(0.4-1.1)$ \\
\hline Halvorsen et al, $2004^{22}$ & 55 & 8.8 & DSM-IV & 0 & 0 \\
\hline Strober et al, $1997^{23}$ & 95 & $10-15^{c}$ & DSM-III & 0 & 0 \\
\hline Wentz et al, $2001^{24}$ & $51^{b}$ & $10^{c}$ & DSM-III-R & 0 & 0 \\
\hline \multicolumn{6}{|c|}{$\begin{array}{l}\text { a. When there is more than one publication on the same study population only the latest is included (Ionger follow-up time). Whenever possible only data on women with anorexia } \\
\text { nevrosa are presented. } \\
\text { b. Study based on the general population, all other studies are of hospitalised patients. } \\
\text { c. Actual follow-up not mean follow-up. } \\
\text { d. } 95 \% \mathrm{Cl} \text { not provided by the authors, thus estimated here when possible on the assumption that the observed number of deaths in each group followed a Poisson distribution. }\end{array}$} \\
\hline
\end{tabular}

disorder, a separate hospitalisation for that disorder was required. All somatic hospitalisations except for those concerning complications of pregnancy, childbirth and the puerperium, were taken into account as somatic comorbidities. Women with admissions because of anorexia alone were considered to have no comorbid conditions.

\section{Follow-up}

The national registration numbers were used to follow-up participants by record linkage to the Swedish Cause-of-Death Register. The time of follow-up was calculated from the date of discharge after the first admission for anorexia nervosa until the date of death or until the end of the study period (31 December 2003). For those with a diagnosis of anorexia who had previously been admitted to hospital for anorexia-related diagnoses (anorexia (ICD-8 code 784.00 and ICD-9 code 783A), vomiting associated with other psychological disturbances (ICD-10 code F50.5), other eating disorders (F50.8) and unspecified eating disorder (F50.9)) the observation period started after discharge for that anorexia-related diagnosis, which is thought to indicate probable anorexia nervosa pathology (anorexia nervosa diagnosis was established afterwards).

\section{Statistics}

The number of expected deaths was calculated by multiplying the number of person-years at risk by 5 -year age group and calendar year-specific mortality rates in the general population. These annual gender-specific mortality rates for different ICD codes were obtained from the official statistics of the Swedish Causeof-Death Register. The SMR, the ratio of observed to expected number of deaths, was used as a measure of risk. The 95\% confidence interval (CI) of the SMR was then calculated on the assumption that the observed number of deaths in each group followed a Poisson distribution.

The effect of specific factors on the fatal outcome of anorexia was tested using a Poisson multiple regression core model and alternatively introduced variables for the control of possible confounders. The SAS statistical package was used for these analyses as well as for simple descriptive and correlation analyses of our data. The study was approved by the ethical committee of the Karolinska Institute.

\section{Results}

\section{Sample characteristics}

A total of 6009 females with at least one admission with an anorexia nervosa diagnosis during the period 1973-2003 were included in the study. Almost $90 \%$ had anorexia nervosa as the main diagnosis in at least one admission. Among the remaining $10 \%$ for which the diagnosis of anorexia was secondary, $56 \%$ received main diagnoses for both somatic and psychiatric disorders during the follow-up period, $30 \%$ received a main diagnosis of a somatic disorder alone, and $10 \%$ received a main diagnosis of 
psychiatric disorder alone. Within the subgroup with anorexia as a secondary diagnosis, $27 \%$ of all admissions with a somatic or a psychiatric diagnosis as a main diagnosis attempted suicide.

Mean age at first admission was 19.4 years (s.d. $=6.3$ ). The duration of the first hospitalisation varied from 0 days to 3 years (1101 days) (mean duration 35.7 days (s.d.=60.4) median=15), and the follow-up period varied from 0 to 31 years (mean follow-up time 13.4 years (s.d.=8.4) median=12.9). The number of admissions for anorexia nervosa varied from 1 to 66 (mean $=3.3$ (s.d.=4.7) median=2), while admissions for other psychiatric and somatic diagnoses varied from 0 to $217($ mean $=3.9 \quad($ s.d. $=11.9)$ median=0) and from 0 to 123 (mean=3.6 (s.d.=7.1) median=1) respectively.

Figure 1 presents the incidence of in-patient-treated anorexia nervosa (mean incidence 4.9 per 100000 women, s.d. $=0.58$ ) and the mean duration of the first hospital stay for a selected period from 1987 to 2003 (data from The Swedish Association of Local Authorities and Regions). ${ }^{33}$ The initial year of this period was 1987, since the Swedish Hospital Discharge Register then covered the whole Swedish population. There was no statistically significant time trend either for incidence or for the mean duration of hospital stay (Spearman correlation coefficient $-0.01 \quad(P=0.97)$ and $0.20(P=0.43)$ respectively).

Table 2 presents the distribution of the study population and the observed deaths across related variables (for a more detailed version, see online Table DS2). More than half (53\%) of the study population was not admitted to hospital for a psychiatric disease, excluding anorexia nervosa, during the study's follow-up period.

\section{Causes of death}

The number of observed deaths was 265. Of these, 126 were as a result of unnatural causes and 53 as a result of mental diseases (online Table DS3). The most frequent main cause of death was suicide $(n=84)$, followed by anorexia nervosa $(n=39)$ and cancer $(n=29)$. Anorexia nervosa was also registered as a secondary cause of death for 30 individuals with a natural main cause of death (in 24 of these anorexia nervosa was even registered as the main cause of death) as well as in 5 individuals with an unnatural main cause of death (online Table DS3). Overall, anorexia nervosa was registered as a main or secondary cause of death on the death certificates of 50 out of 265 people (19\%). The mean age at death was 34.2 years $($ s.d. $=10.7)$.

\section{Suicide as a cause of death}

Among people who died by suicide, 41 used a non-violent method (self-poisoning with drugs in 39 cases and gas poisoning in 2 cases) and 41 chose a violent method including hanging $(n=17)$, drowning $(n=8)$, jumping from high places or in front of a moving vehicle $(n=9)$, using sharp objects $(n=5)$ and 2 were registered as 'intentional self-harm by other specified means'.

\section{Standardised mortality ratios}

Standardised mortality ratios for the unnatural causes of death were calculated on the basis of 80388 person-years of follow-up. The follow-up time for the natural causes of death was 74523 person-years (1-year delay in the beginning of follow-up).

Table 3 presents the SMR for different causes of death according to the ICD-9 diagnostic categories. The SMR for all causes of death was 6.2 (95\% CI 5.5-7.0). The SMR was significantly increased for almost all groups of natural and unnatural causes of death. Anorexia nervosa had the highest SMR at 650.0 (95\% CI 462.2-888.6), psychoactive substance use had the second highest at 18.9 (95\% CI 10.0-32.3) and suicide followed with an SMR of 13.6 (95\% CI 10.9-16.8).

The SMR for unnatural causes of death during the first year after the first hospital admission was 19.3 (95\% CI 11.2-30.9), and remained significantly high 20 years or more afterwards at

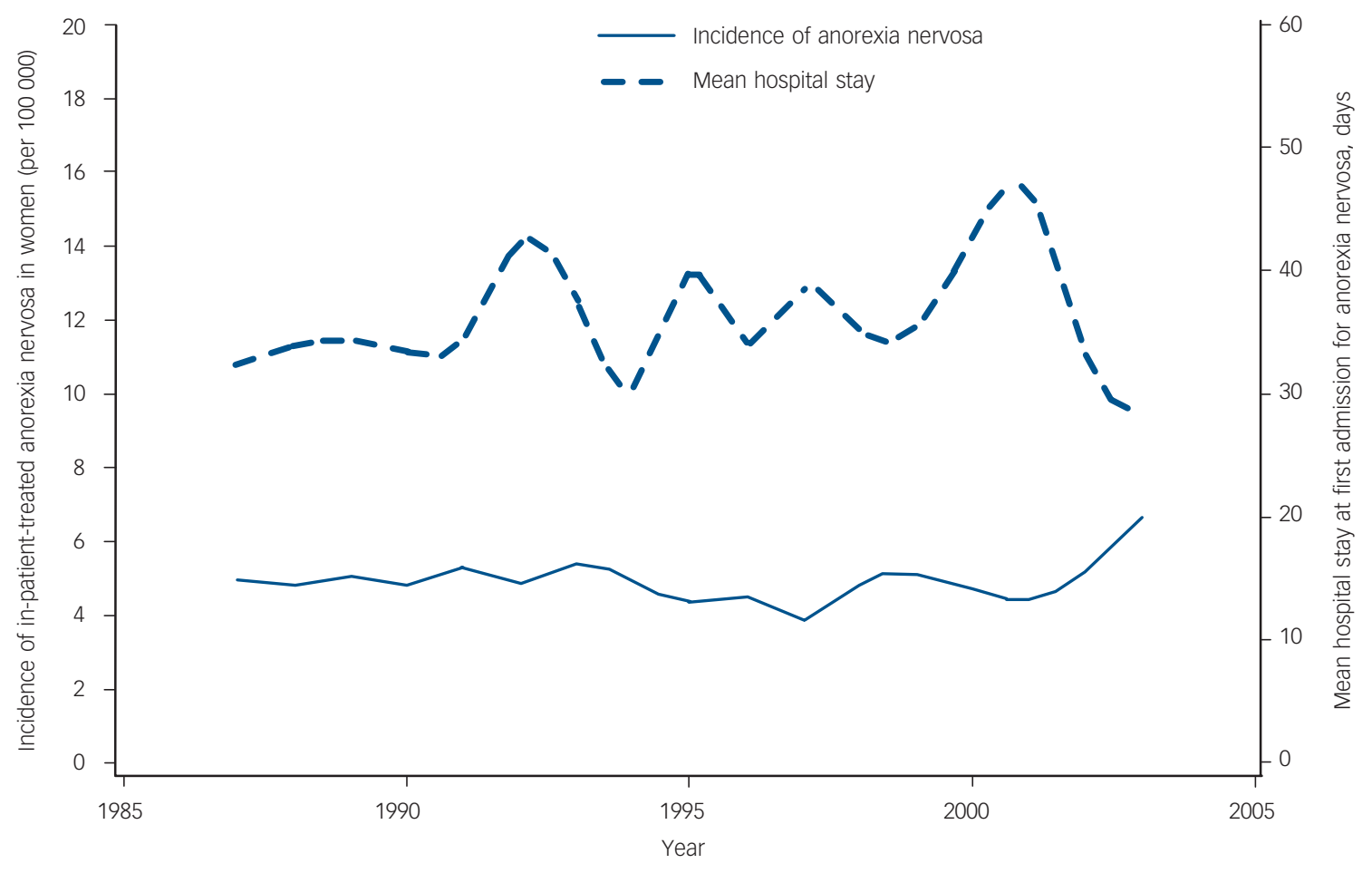

Fig. 1 Incidence of hospitalisations for anorexia nervosa and mean duration of the first hospitalisation over time. 
Table 2 Distribution of population and observed deaths (total, unnatural and natural) across related variables ${ }^{a}$

\begin{tabular}{|c|c|c|c|c|}
\hline & $n(\%)$ & Total deaths, $n(\%)$ & Unnatural deaths, $n(\%)$ & Natural deaths, $n(\%)$ \\
\hline All & 6009 & 265 & 126 & 139 \\
\hline \multicolumn{5}{|c|}{ Age at first anorexia nervosa admission, years } \\
\hline $10-19$ & 3691 (61) & $78(29)$ & 42 (33) & $36(26)$ \\
\hline $20-29$ & $1823(30)$ & $116(44)$ & $58(46)$ & $58(42)$ \\
\hline 30-39 & 495 (8) & $71(27)$ & $26(21)$ & 45 (32) \\
\hline \multicolumn{5}{|l|}{ Hospitalisations, $n$} \\
\hline \multicolumn{5}{|l|}{ Anorexia nervosa } \\
\hline $1-5$ & $5124(85)$ & $176(66)$ & $87(69)$ & $89(64)$ \\
\hline $6-10$ & $554(9)$ & $40(15)$ & $18(14)$ & $22(16)$ \\
\hline $11+$ & $331(6)$ & 49 (18) & $21(17)$ & $28(20)$ \\
\hline \multicolumn{5}{|c|}{ Other psychiatric disorders ${ }^{\mathrm{b}}$} \\
\hline 0 & 3201 (53) & $75(28)$ & $26(21)$ & 49 (35) \\
\hline $1-5$ & $1886(31)$ & $93(35)$ & $46(36)$ & $47(34)$ \\
\hline $6-10$ & $360(6)$ & $28(11)$ & $16(13)$ & $12(9)$ \\
\hline $11+$ & $562(9)$ & $69(26)$ & $38(30)$ & $31(22)$ \\
\hline \multicolumn{5}{|l|}{ Somatic diseases $\mathrm{b}^{\mathrm{c}} \mathrm{c}$} \\
\hline 0 & $1746(29)$ & $38(14)$ & $22(18)$ & $16(12)$ \\
\hline $1-5$ & $3264(54)$ & $115(43)$ & $57(45)$ & $58(42)$ \\
\hline $6-10$ & 540 (9) & 50 (19) & $26(21)$ & $24(17)$ \\
\hline $11+$ & $459(8)$ & $62(23)$ & $21(17)$ & $41(30)$ \\
\hline \multicolumn{5}{|c|}{ Medical history before first admission } \\
\hline No admissions & $3190(53)$ & $126(48)$ & $57(45)$ & $69(50)$ \\
\hline Somatic historyc ${ }^{c}$ & $1963(33)$ & $70(26)$ & $33(26)$ & $37(27)$ \\
\hline Psychiatric history & $266(4)$ & $18(7)$ & $7(6)$ & $11(8)$ \\
\hline Both & $590(10)$ & $51(19)$ & $29(23)$ & $22(16)$ \\
\hline \multicolumn{5}{|c|}{ Comorbidity after first admission } \\
\hline None & $1585(26)$ & $24(9)$ & $12(10)$ & $12(9)$ \\
\hline Somatic $^{c}$ & 1765 (29) & $54(20)$ & $16(13)$ & $38(28)$ \\
\hline Psychiatric & $474(8)$ & $18(7)$ & $13(10)$ & $5(4)$ \\
\hline Both & 2185 (36) & 169 (64) & $85(68)$ & $84(60)$ \\
\hline
\end{tabular}

8.2 (95\% CI 4.2-14.3) (Table 4). The SMR for natural causes of death during the second year after the first hospital admission, which was the first follow-up year for the natural causes of death, was 12.1 (95\% CI 7.5-18.5) and remained significantly high 20 years or more afterwards at 2.7 (95\% CI 1.7-4.0). The overall SMR for people with no admissions for psychiatric diagnoses other than anorexia nervosa during the follow-up was 3.6 (95\% CI 2.8-4.5) (data not shown in tables).

\section{Prognostic factors}

Online Table DS4 presents the effect of possible prognostic factors on the fatal outcome of anorexia controlling for the calendar period of first admission, age and duration of stay for the first admission, age at follow-up and follow-up time.

The mortality for individuals first admitted during 1987-1996 and 1997-2003 was substantially lower (relative risk $(\mathrm{RR})=0.4$, 95\% CI 0.3-0.5 and RR $=0.3,95 \%$ CI $0.2-0.6$ respectively) in comparison with those first admitted during 1973-1979. The difference was more profound with respect to natural causes of death.

Older age at first admission was associated with increased mortality, with a more than twofold increase for those aged 20$29(\mathrm{RR}=2.2,95 \% \mathrm{CI} 1.5-3.3)$ and an almost fourfold increase for those aged 30-39 ( $R R=3.6,95 \%$ CI 2.0-6.6) compared with those aged 10-19 at first admission.

Longer duration of the first hospital stay was associated with lower mortality. Those individuals who had received in-patient care at the first admission lasting more than 1 month had therefore a more favourable outcome ( $\mathrm{RR}=0.7$, 95\% CI $0.5-0.9)$ compared with those who only stayed for up to 5 days, but this difference did not reach statistical significance with respect to the unnatural causes of death.

There was a trend towards lower mortality over the follow-up time, although this did not reach statistical significance with the exception of a more than twofold increase during the second year following discharge after the first hospitalisation for anorexia $(\mathrm{RR}=2.6,95 \% \mathrm{CI} 1.2-5.5)$, compared with mortality 20 years or more after the first hospitalisation.

Repeated admissions for anorexia were associated with higher mortality. Those women admitted to hospital six to ten times, or more than ten times, had worse outcomes ( $\mathrm{RR}=2.0$, 95\% CI $1.4-$ 2.8 and $\mathrm{RR}=3.6,95 \%$ CI 2.6-5.0 respectively) compared with those who were admitted one to five times.

Hospitalisations for other psychiatric and somatic disorders were found to worsen the outcome of anorexia. Repeated admissions (more than ten) for psychiatric disorders had a profound effect on the risk of death from unnatural causes ( $R R=6.4,95 \%$ CI 3.8-10.7), although repeated admissions for somatic disorders had a more profound effect on the risk of death from natural causes $(\mathrm{RR}=4.0,95 \% \mathrm{CI} 2.2-7.3)$.

After the first admission for anorexia nervosa, psychiatric comorbidity in terms of in-patient care for psychiatric disorders was associated with a threefold increased risk of death from unnatural causes ( $\mathrm{RR}=3.0,95 \%$ CI 1.4-6.5) compared with no comorbidity, while both psychiatric and somatic comorbidity were associated with a worse outcome concerning both unnatural and natural causes of death $(\mathrm{RR}=3.1,95 \% \mathrm{CI} 1.7-5.7$ and $\mathrm{RR}=2.4$, 95\% CI 1.3-4.4 respectively). 


\begin{tabular}{|c|c|c|c|c|}
\hline Underlying cause of death & Observed deaths, $n$ & Expected deaths, $n$ & $S M R^{a}$ & $(95 \% \mathrm{Cl})$ \\
\hline Total & 265 & 42.7 & 6.2 & $(5.5-7.0)$ \\
\hline Natural & 139 & 28.5 & 4.9 & $(4.1-5.8)$ \\
\hline Infections & 0 & 0.6 & 0.01 & $(0-6.5)$ \\
\hline Cancer & 29 & 15.1 & 1.9 & $(1.3-2.8)$ \\
\hline Endocrine & 9 & 1.1 & 7.9 & $(3.6-14.9)$ \\
\hline Haematopoietic & 1 & 0.1 & 7.8 & $(0.2-43.2)$ \\
\hline Mental & $54^{\mathrm{b}}$ & 0.9 & 62.8 & $(47.2-81.9)$ \\
\hline Psychoactive substance use & 13 & 0.7 & 18.9 & $(10.0-32.3)$ \\
\hline Anorexia nervosa & 39 & 0.1 & 650.0 & $(462.2-888.6)$ \\
\hline Nervous system & 4 & 1.5 & 2.6 & $(0.7-6.6)$ \\
\hline Cardiovascular & 11 & 4.8 & 2.3 & $(1.1-4.1)$ \\
\hline Respiratory & 14 & 1.2 & 11.5 & (6.3-19.3) \\
\hline Gastrointestinal & 6 & 1.1 & 5.4 & $(2.0-11.7)$ \\
\hline Urogenital & 2 & 0.2 & 10.8 & $(1.3-38.8)$ \\
\hline Dermatological & 0 & 0.0 & - & \\
\hline Autoimmune & 3 & 0.3 & 8.8 & $(1.8-25.7)$ \\
\hline Other & 6 & 1.5 & 3.9 & $(1.4-8.5)$ \\
\hline Unnatural & 126 & 14.2 & 8.9 & $(7.4-10.6)$ \\
\hline Suicide & 84 & 6.2 & 13.6 & $(10.9-16.8)$ \\
\hline Homicide & 4 & 0.8 & 5.2 & $(1.4-13.4)$ \\
\hline Traffic accidents & 7 & 3.9 & 1.8 & $(0.7-3.7)$ \\
\hline Undefined & 19 & 1.7 & 10.9 & $(6.6-17.1)$ \\
\hline Other & 12 & 1.6 & 7.5 & $(3.9-13.0)$ \\
\hline
\end{tabular}

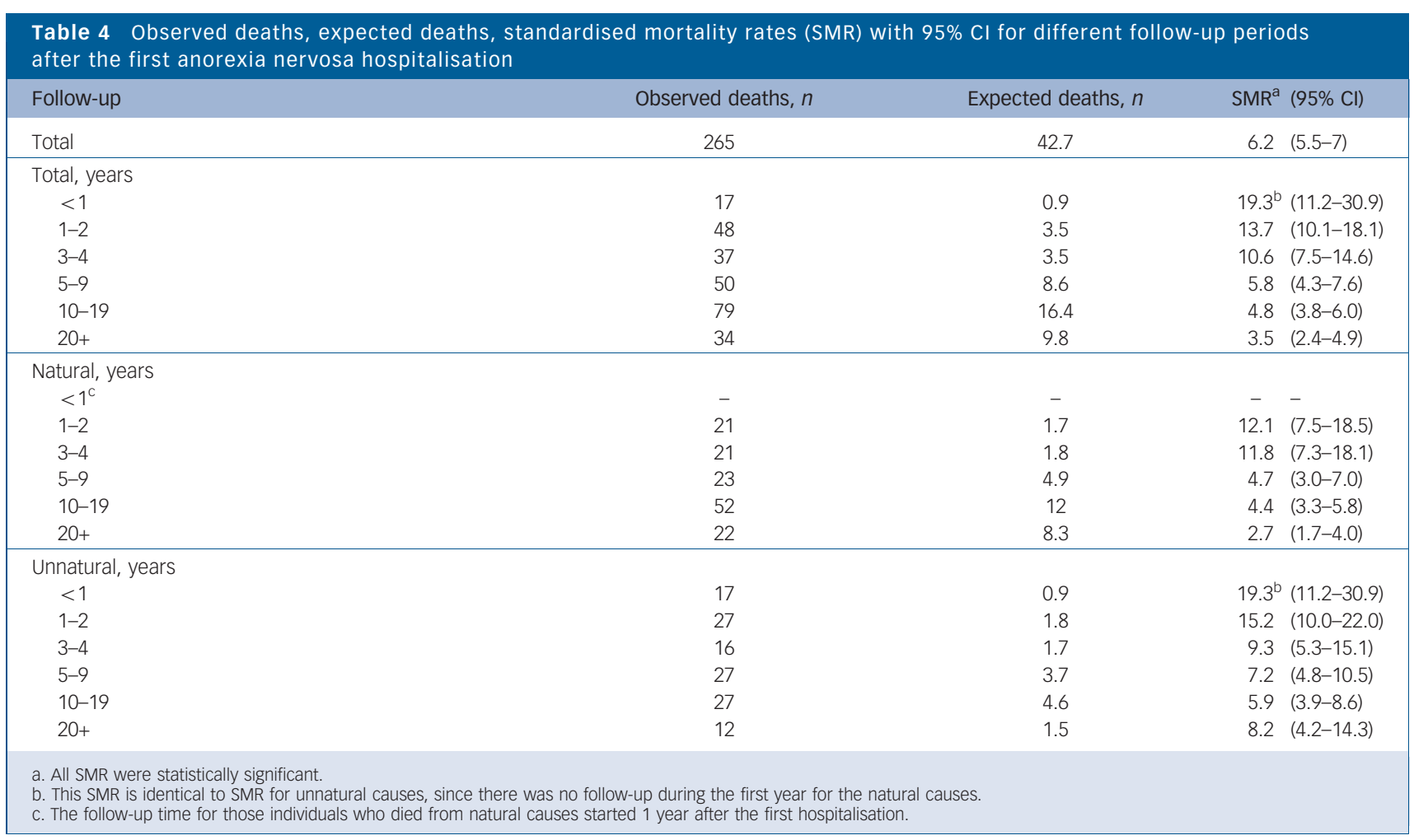

\section{Discussion}

Mortality from anorexia nervosa has been extensively studied. The substantial variation that is seen in the SMR reported elsewhere $\left(0-17.8^{1-24}\right)$ can be explained at least in part by methodological shortcomings such as heterogeneity of the inclusion criteria and study population (general population $v$. hospitalised patients), diversity of the sample size (48-2763 individuals) and length of mean follow-up time (6-36.2 years), and differences in the standardisation method, but also by the treatment offered. Overall, people with anorexia nervosa in this cohort had a sixfold increased mortality compared with the general population. The SMR for natural as well as for unnatural causes of death remained significantly high even 20 years or more after the first hospitalisation for anorexia. 
The highest SMR was indeed found for anorexia. Anorexia nervosa as a main or secondary cause of death was stated in the death certificates of almost $20 \%$ of the cases. This is logically expected, given the high mortality of anorexia soon after its presentation, but also the chronicity of this disorder with approximately $20 \%$ of the people becoming chronically ill. ${ }^{34}$

The second highest SMR was for psychoactive substance use (18.9). This finding represents mainly alcohol-related diseases rather than illicit drug use; 10 out of 13 deaths were alcohol-related. Lifetime alcohol dependence has been found to be over-represented in women with anorexia nervosa ${ }^{35}$ and alcohol use at follow-up has been shown to be one of the strongest and most consistent predictors of fatal outcome. ${ }^{3}$ Furthermore, the fivefold mortality increase in gastrointestinal diseases may also be because of, at least in part, indirect effects of alcohol use, since four out of the six deaths in this category were from liver cirrhosis. It has been reported that a third of women with anorexia nervosa who develop alcohol dependence do not have alcohol-related problems at the beginning of their anorexia course, ${ }^{3}$ which indicates the need to provide better assessment of such disorders during follow-up.

The stable finding that suicide accounts for approximately 20$30 \%$ of the deaths from anorexia nervosa ${ }^{36,37}$ was also confirmed in the present study. The estimated SMR for suicide (13.6) is lower than figures reported elsewhere, which range from 23.1 to 56.9 .,38 Alcohol misuse is a recognised risk factor for suicide $e^{38,39}$ and is also found to be over-represented in women with anorexia who also have a history of suicide attempts. ${ }^{40}$ The indirect impact of thorough assessment and treatment of alcohol-related disorders in relation to suicide in this population should be considered in prevention strategies.

Women in this cohort had 11 and 10 times higher death rates from diseases in respiratory and urogenital systems respectively. In 12 out of 16 deaths in these systems, the cause of death was infectious diseases (pneumonia and pyelonephritis). Reduced fever response and fewer signs and symptoms of infection can significantly delay diagnosis of an infection in people with anorexia nervosa, thereby increasing complication rates. ${ }^{41}$ Some deaths due to pneumonia may also be attributable to alcoholism. ${ }^{42}$

The relation between diabetes and eating disorders has been studied extensively. A higher prevalence of bulimia nervosa but not anorexia nervosa was found among people with type 1 diabetes in a recent meta-analysis. ${ }^{43}$ Mortality in this subgroup of individuals with concurrent anorexia and diabetes type 1 has been estimated to be 14 times higher than expected. ${ }^{44}$ Early and severe neurovascular complications have been reported in people with concurrent eating disorders and insulin dependent diabetes, partly as a result of dangerous practices such as insulin misuse. ${ }^{45}$ In our cohort, an almost eightfold increase in mortality was found for endocrine causes of death, with diabetes over-represented.

A fivefold increased mortality from homicide in our study is in line with the increased risk of being a victim of homicide reported among women with mental disorders in general. ${ }^{46}$

A modest excess mortality from cardiovascular diseases for all psychiatric diseases in women $(\mathrm{SMR}=1.36)$ has been reported, ${ }^{47}$ and other reports on bipolar and unipolar disorder ${ }^{48}$ as well as on schizophrenia ${ }^{49}$ in women suggest an almost twofold higher risk. In our cohort, a twofold increased risk for death from cardiovascular causes was estimated. One study has reported a lower than expected mortality from cardiovascular diseases in people with anorexia, ${ }^{21}$ possibly mediated by the protective effect of low energy intake and/or excessive physical activity in this population. Other risk factors for cardiovascular diseases such as hypercholesterolaemia, which is common in individuals with anorexia, ${ }^{50}$ smoking and alcohol misuse could explain a higher risk. Moreover, some of this excess mortality can stem from direct cardiovascular complications, especially during the acute treatment of anorexia nervosa. ${ }^{51}$

The twofold increase in cancer mortality in our study is intriguing. Reports of cancer among women with anorexia nervosa have been limited. ${ }^{21,26,27}$ In one case a statistically significant lower incidence rate for breast cancer was reported. ${ }^{26}$ Other reports on cancer mortality among psychiatric patients show a slightly increased cancer risk in women with bipolar disorder and schizophrenia $\left(\mathrm{SMR}=1.2^{48}\right.$ and $\mathrm{SMR}=1.3^{49}$ respectively), although other studies have not found significant differences, ${ }^{47,52}$ possibly because of lack of statistical power. The doubled cancer mortality in our cohort is noteworthy. The extent to which known risk factors for cancer, such as smoking and alcohol misuse, overcome protective factors such as physical activity and low oestrogen levels (for some cancers), as well as the role of severe and chronic malnutrition in human carcinogenesis must be further elucidated. Research suggests an increased prevalence of smoking among women with certain subtypes of anorexia (binging and/or purging), as well as among women with a history of bulimia, but not in restrictive anorexia nervosa. ${ }^{53}$

Regarding time trends in our cohort, there was no significant change in yearly incidence of in-patient-treated anorexia nervosa or in mean hospital stay at the first admission over time from 1987 to 2003. During this period, the number of hospital beds in Sweden was drastically reduced by approximately $75 \%$. The mean hospital stay for psychiatric disorders in the same age group was reduced by more than $30 \%{ }^{33}$ The fact that anorexia has been an exception to this rule may be because of the immediate high risk for fatal outcome in people with anorexia nervosa, as a consequence of somatic complications and increased suicidality.

The lower overall mortality from anorexia during more recent years confirms the results of another Swedish study ${ }^{11}$ with partly overlapping data. Advances in the medical treatment of individuals with acute anorexia nervosa, with better refeeding strategies and better control of the medical complications, as well as advances in treatment of the psychological aspects of the disease or better treatment of psychiatric comorbidities, may account for the decrease in mortality from both natural and unnatural causes of death. ${ }^{35}$

The impact of duration of in-patient treatment has been investigated in many studies but is considered unclear because of ambiguous findings. ${ }^{34}$ In our study, in-patient treatment of 1 month or longer at first admission for anorexia was accompanied by significantly lower mortality. This may be explained by a more systematic and broader therapeutic approach where both somatic and psychological issues are taken into consideration during longer hospital stays. Care in psychiatric settings was indeed found to be associated with longer hospital stay in our data (not shown in tables).

In another study, follow-up time has been found to correlate negatively with SMR, with the latter having a peak of 30 the first year after presentation but remaining significantly high for up to 15 years after presentation. ${ }^{54}$ In our sample, a very high SMR was also found for the first year after first hospitalisation for anorexia and the SMR was still significantly high 20 years or more after the first hospitalisation.

Psychiatric comorbidity is common in anorexia nervosa. ${ }^{34}$ More than half of our population were not hospitalised for other psychiatric disorders during follow-up. After first hospitalisation, in-patient care for psychiatric disorders tripled the mortality from unnatural causes, while hospital care for both psychiatric and somatic diseases increased the mortality from natural causes more than twofold. 


\section{Strengths and limitations}

This is the first time that mortality from both natural and unnatural causes of death has been studied in detail in anorexia nervosa. Moreover, this study includes the largest number of people with anorexia published so far. By using a homogeneous large nationwide sample of in-patients and a long follow-up period, the statistical power was adequate for the investigation of mortality patterns and potential prognostic factors in this disease.

However, a number of limitations must be taken into consideration when interpreting the findings of this study. This is a register-based retrospective cohort study that relies only on in-patient treatment of anorexia nervosa and therefore probably addresses the most severe cases of the disease. These findings may not represent the outcome of anorexia in out-patients or even more profoundly in those individuals with the disease who never seek help and therefore remain outside the treatment facilities. The same applies to psychiatric and somatic comorbidity; they are defined as receiving in-patient care and thus represent a more severe form of comorbidity. Since the definitions of eating disorders in the ICD-8 classification system are rather diffuse, the possibility that individuals with bulimia are included in our sample cannot be excluded. Although this was not a primary aim of our study, we examined a subset of this cohort consisting of women with an in-patient diagnosis of anorexia nervosa from 1987 onwards (when a possible bulimia nervosa diagnosis was introduced along with ICD-9). In this subset, 502 women (14\%) were admitted on a separate occasion with a bulimia diagnosis during follow-up (data not shown). This finding could either reflect a lower diagnostic validity of earlier versions of ICD with regard to anorexia or the natural course of anorexia itself with the not uncommon subsequent development of bulimia symptoms. However, the relatively stable incidence of in-patienttreated anorexia over time suggests a smooth integration of different ICD systems as far as the diagnosis of the disease is concerned in Sweden. Moreover, diagnostic misclassification of other somatic diagnoses as anorexia nervosa cannot be excluded, but our inclusion criteria were specifically selected to reduce this possibility. Information on the disease's onset, and therefore on the duration of the illness before first hospitalisation, was not available.

In conclusion, this study addresses the severity of anorexia nervosa in a large sample of in-patient-treated Swedish women. The increased mortality from both natural and unnatural causes of death that persists throughout their lifetime is astonishing. More research is needed on the specific risk factors that bring about this unfavourable outcome so that better treatment strategies can be offered in the future. However, assessment and treatment of psychiatric comorbidity, especially alcohol misuse, may be a pathway to a better long-term outcome.

Fotios C Papadopoulos, MD, PhD, Department of Neuroscience, Psychiatry, University Hospital, Uppsala; Anders Ekbom, MD, PhD, Lena Brandt, BSc, Unit of Clinical Epidemiology, Department of Medicine, Karolinska Hospital, Karolinska Institute, Stockholm; Lisa Ekselius, MD, PhD, Department of Neuroscience, Psychiatry, University Hospital, Uppsala, Sweden.

Correspondence: Fotios Papadopoulos, Department of Neuroscience, Psychiatry Uppsala University Hospital, SE-751 85 Uppsala, Sweden. Email: fotis.papadopoulos@gmail.com

First received 12 Feb 2008, final revision 9 May 2008, accepted 9 Jul 2008

\section{Acknowledgements}

The work of F.C.P. and L.E. was in part financially supported by the Swedish Research council.

\section{References}

1 Norring CE, Sohlberg SS. Outcome, recovery, relapse and mortality across six years in patients with clinical eating disorders. Acta Psychiatr Scand 1993; 87: $437-44$

2 Eckert ED, Halmi KA, Marchi P, Grove W, Crosby R. Ten-year follow-up of anorexia nervosa: clinical course and outcome. Psychol Med 1995; 25 143-56.

3 Keel PK, Dorer DJ, Eddy KT, Franko D, Charatan DL, Herzog DB. Predictors of mortality in eating disorders. Arch Gen Psychiatry 2003; 60: 179-83.

4 Lee S, Chan YY, Hsu LK. The intermediate-term outcome of Chinese patients with anorexia nervosa in Hong Kong. Am J Psychiatry 2003; 160: 967-72.

5 Birmingham CL, Su J, Hlynsky JA, Goldner EM, Gao M. The mortality rate from anorexia nervosa. Int J Eat Disord 2005; 38: 143-6.

6 Lowe B, Zipfel S, Buchholz C, Dupont Y, Reas DL, Herzog W. Long-term outcome of anorexia nervosa in a prospective 21-year follow-up study. Psychol Med 2001; 31: 881-90.

7 Signorini A, De Filippo E, Panico S, De Caprio C, Pasanisi F, Contaldo F. Longterm mortality in anorexia nervosa: a report after an 8-year follow-up and a review of the most recent literature. Eur J Clin Nutr 2007; 61: 119-22.

8 Moller-Madsen S, Nystrup J, Nielsen S. Mortality in anorexia nervosa in Denmark during the period 1970-1987. Acta Psychiatr Scand 1996; 94: 454-9.

9 Fichter MM, Quadflieg N, Hedlund S. Twelve-year course and outcome predictors of anorexia nervosa. Int J Eat Disord 2006; 39: 87-100.

10 Crow S, Praus B, Thuras P. Mortality from eating disorders - a 5- to 10-year record linkage study. Int J Eat Disord 1999; 26: 97-101.

11 Lindblad F, Lindberg L, Hjern A. Improved survival in adolescent patients with anorexia nervosa: a comparison of two Swedish national cohorts of female inpatients. Am J Psychiatry 2006; 163: 1433-5.

12 Joergensen J. The epidemiology of eating disorders in Fyn County, Denmark, 1977-1986. Acta Psychiatr Scand 1992; 85: 30-4

13 Pagsberg AK, Wang AR. Epidemiology of anorexia nervosa and bulimia nervosa in Bornholm County, Denmark, 1970-1989. Acta Psychiatr Scand 1994; 90: 259-65.

14 Emborg C. Mortality and causes of death in eating disorders in Denmark 1970-1993: a case register study. Int J Eat Disord 1999; 25: 243-51.

15 Patton GC. Mortality in eating disorders. Psychol Med 1988; 18: 947-51.

16 Tolstrup K, Brinch M, Isager T, Nielsen S, Nystrup J, Severin B, et al. Longterm outcome of 151 cases of anorexia nervosa. The Copenhagen Anorexia Nervosa Follow-Up Study. Acta Psychiatr Scand 1985; 71: 380-7.

17 Crisp AH, Callender JS, Halek C, Hsu LK. Long-term mortality in anorexia nervosa. A 20-year follow-up of the St George's and Aberdeen cohorts. Br J Psychiatry 1992; 161: 104-7.

18 Millar HR, Wardell F, Vyvyan JP, Naji SA, Prescott GJ, Eagles JM. Anorexia nervosa mortality in Northeast Scotland, 1965-1999. Am J Psychiatry 2005; 162: $753-7$.

19 Theander S. Anorexia nervosa. A psychiatric investigation of 94 female patients. Acta Psychiatr Scand Suppl 1970; 214: 1-194.

20 Crisp AH. Death, survival and recovery in anorexia nervosa: a thirty five year study. Eur Eat Disorders Rev 2006; 14: 168-75.

21 Korndorfer SR, Lucas AR, Suman VJ, Crowson CS, Krahn LE, Melton LJ, 3rd. Long-term survival of patients with anorexia nervosa: a population-based study in Rochester, Minn. Mayo Clin Proc 2003; 78: 278-84.

22 Halvorsen I, Andersen A, Heyerdahl S. Good outcome of adolescent onset anorexia nervosa after systematic treatment. Intermediate to long-term follow-up of a representative county-sample. Eur Child Adolesc Psychiatry 2004; 13: 295-306.

23 Strober M, Freeman R, Morrell W. The long-term course of severe anorexia nervosa in adolescents: survival analysis of recovery, relapse, and outcome predictors over 10-15 years in a prospective study. Int J Eat Disord 1997; 22: 339-60.

24 Wentz E, Gillberg C, Gillberg IC, Rastam M. Ten-year follow-up of adolescentonset anorexia nervosa: psychiatric disorders and overall functioning scales. J Child Psychol Psychiatry 2001; 42: 613-22.

25 Herzog DB, Greenwood DN, Dorer DJ, Flores AT, Ekeblad ER, Richards A, et al. Mortality in eating disorders: a descriptive study. Int J Eat Disord 2000; 28: 20-6.

26 Michels KB, Ekbom A. Caloric restriction and incidence of breast cancer JAMA 2004; 291: 1226-30.

27 Mellemkjaer L, Emborg C, Gridley G, Munk-Jorgensen P, Johansen C, Tjonneland $\mathrm{A}$, et al. Anorexia nervosa and cancer risk. Cancer Causes Control 2001; 12: 173-7. 
28 National Board of Health and Welfare. Causes of Death 2004. National Board of Health and Welfare, 2007.

29 National Board of Health and Welfare. The Swedish Hospital Discharge Register 2007 National Board of Health and Welfare (http:// www.socialstyrelsen.se/en/Statistics/statsbysubject/The+Swedish+Hospital+Discharge+Register.htm).

30 World Health Organization. International Statistical Classification of Diseases and Related Health Problems (ICD-8). WHO, 1967.

31 World Health Organization. International Statistical Classification of Diseases and Related Health Problems (ICD-9). WHO, 1978.

32 World Health Organization. The ICD-10 Classification of Mental and Behavioural Disorders: Clinical Descriptions and Diagnostic Guidelines. WHO, 1992.

33 The Swedish Association of Local Authorities and Regions (http:// sjvdata.skl.se/sif/start/)

34 Steinhausen HC. The outcome of anorexia nervosa in the 20th century. Am J Psychiatry 2002; 159: 1284-93.

35 Sullivan PF, Bulik CM, Fear JL, Pickering A. Outcome of anorexia nervosa: a case-control study. Am J Psychiatry 1998; 155: 939-46.

36 Sullivan PF. Mortality in anorexia nervosa. Am J Psychiatry 1995; 152: 1073-4.

37 Harris EC, Barraclough B. Excess mortality of mental disorder. Br J Psychiatry 1998; 173: 11-53.

38 Harris EC, Barraclough B. Suicide as an outcome for mental disorders. A meta-analysis. Br J Psychiatry 1997; 170: 205-28.

39 McGirr A, Seguin M, Renaud J, Benkelfat C, Alda M, Turecki G. Gender and risk factors for suicide: evidence for heterogeneity in predisposing mechanisms in a psychological autopsy study. J Clin Psychiatry 2006; 67 1612-7.

40 Favaro A, Santonastaso P. Suicidality in eating disorders: clinical and psychological correlates. Acta Psychiatr Scand 1997; 95: 508-14.

41 Brown RF, Bartrop R, Beumont $\mathrm{P}$, Birmingham CL. Bacterial infections in anorexia nervosa: delayed recognition increases complications. Int J Eat Disord 2005; 37: 261-5.
42 Zilber N, Schufman N, Lerner Y. Mortality among psychiatric patients - the groups at risk. Acta Psychiatr Scand 1989; 79: 248-56.

43 Mannucci E, Rotella F, Ricca V, Moretti S, Placidi GF, Rotella CM. Eating disorders in patients with type 1 diabetes: a meta-analysis. $J$ Endocrinol Invest 2005; 28: 417-9.

44 Nielsen S, Emborg C, Molbak AG. Mortality in concurrent type 1 diabetes and anorexia nervosa. Diabetes Care 2002; 25: 309-12.

45 Peveler RC, Bryden KS, Neil HA, Fairburn CG, Mayou RA, Dunger DB, et al. The relationship of disordered eating habits and attitudes to clinical outcomes in young adult females with type 1 diabetes. Diabetes Care 2005; 28: 84-8.

46 Hiroeh U, Appleby L, Mortensen PB, Dunn G. Death by homicide, suicide, and other unnatural causes in people with mental illness: a population-based study. Lancet 2001; 358: 2110-2.

47 Joukamaa $\mathrm{M}$, Heliövaara $\mathrm{M}$, Knekt $\mathrm{P}$, Aromaa $\mathrm{A}$, Raitasalo $\mathrm{R}$, Lehtinen V. Mental disorders and cause-specific mortality. Br J Psychiatry 2001; 179: 498-502.

48 Osby U, Brandt L, Correia N, Ekbom A, Sparen P. Excess mortality in bipolar and unipolar disorder in Sweden. Arch Gen Psychiatry 2001; 58: 844-50.

49 Osby U, Correia N, Brandt L, Ekbom A, Sparen P. Mortality and causes of death in schizophrenia in Stockholm county, Sweden. Schizophr Res 2000; 45: $21-8$

50 Klinefelter HF. Hypercholesterolemia in anorexia nervosa. J Clin Endocrinol Metab 1965; 25: 1520-1.

51 Casiero D, Frishman WH. Cardiovascular complications of eating disorders. Cardiol Rev 2006; 14: 227-31.

52 Stark C, MacLeod M, Hall D, O'Brien F, Pelosi A. Mortality after discharge from long-term psychiatric care in Scotland, 1977-94: a retrospective cohort study. BMC Public Health 2003; 3: 30.

53 Anzengruber D, Klump KL, Thornton L, Brandt H, Crawford S, Fichter MM, et al. Smoking in eating disorders. Eat Behav 2006; 7: 291-9.

54 Nielsen S, Moller-Madsen S, Isager T, Jorgensen J, Pagsberg K, Theander S. Standardized mortality in eating disorders - a quantitative summary of previously published and new evidence. J Psychosom Res 1998; 44: $413-34$ 